\title{
Change detection in quad and dual pol, single- and bi-frequency SAR data
}

\author{
Nielsen, Allan Aasbjerg; Conradsen, Knut; Skriver, Henning
}

Published in:

Proceedings of SPIE Image and Signal Processing for Remote Sensing XXI

Link to article, DOI:

$10.1117 / 12.2194853$

Publication date:

2015

Document Version

Publisher's PDF, also known as Version of record

Link back to DTU Orbit

Citation (APA):

Nielsen, A. A., Conradsen, K., \& Skriver, H. (2015). Change detection in quad and dual pol, single- and bifrequency SAR data. In L. Bruzzone (Ed.), Proceedings of SPIE Image and Signal Processing for Remote Sensing XXI [964313] SPIE - International Society for Optical Engineering. Proceedings of SPIE - The International Society for Optical Engineering https://doi.org/10.1117/12.2194853

\section{General rights}

Copyright and moral rights for the publications made accessible in the public portal are retained by the authors and/or other copyright owners and it is a condition of accessing publications that users recognise and abide by the legal requirements associated with these rights.

- Users may download and print one copy of any publication from the public portal for the purpose of private study or research.

- You may not further distribute the material or use it for any profit-making activity or commercial gain

- You may freely distribute the URL identifying the publication in the public portal 


\title{
Change detection in quad and dual pol, single- and bi-frequency SAR data
}

\author{
Allan A. Nielsen ${ }^{a}$, Knut Conradsen ${ }^{a}$ and Henning Skriver ${ }^{b}$ \\ ${ }^{a}$ Technical University of Denmark \\ DTU Compute - Applied Mathematics and Computer Science \\ DK-2800 Kgs. Lyngby, Denmark \\ ${ }^{b}$ Technical University of Denmark \\ DTU Space - National Space Institute \\ DK-2800 Kgs. Lyngby, Denmark
}

\begin{abstract}
When the covariance matrix representation is used for multi-look polarimetric synthetic aperture radar (SAR) data, the complex Wishart distribution applies. Based on this distribution a likelihood ratio test statistic for equality of two complex variance-covariance matrices and an associated $p$-value are given. In a case study airborne EMISAR C- and L-band SAR images covering agricultural fields and wooded areas near Foulum, Denmark, are used in single- and bi-frequency, bi-temporal change detection with full and dual polarimetry data.
\end{abstract}

\section{INTRODUCTION}

In 2003 we introduced a likelihood ratio test statistic for equality of two variance-covariance matrices following the complex Wishart distribution with an associated $p$-value. ${ }^{1}$ We demonstrated their use to change detection in fully polarimetric (quad pol) SAR data. We also showed the possibility of application of the test statistic and the associated $p$-value to block-structured covariance SAR data such as the azimuthal symmetric and diagonal-only cases. We elaborated on this feature and we also showed the application of the test statistic and the associated $p$-value to edge detection in polarimetric SAR data. ${ }^{2}$ Later, we introduced multi-frequency applications. ${ }^{3}$ Also the Hotelling-Lawley trace statistic has been applied to the change detection problem. ${ }^{4}$

In the early 2000s, not many workers had access to polarimetric SAR data. With the advent of several spaceborne polarimetric SAR instruments this situation has improved. These instruments include

- the Japanese ALOS (a.k.a. DAICHI), L-band with single, dual and full polarization,

- the Canadian Radarsat-2, C-band with single, dual and full polarization,

- the German TerraSAR-X, X-band with single, dual and full polarization,

- the Italian COSMO-SkyMed, X-band with single and dual polarization,

- the European (ESA) Sentinel-1, C-band with single and dual polarization.

For power supply and coverage reasons some of the instruments among the above which have full polarization capability are often operated in (either single or) dual polarization modes, i.e., they transmit one polarization only and receive (either one or) both polarizations. A revisit to the methods described and sketched earlier ${ }^{1-3}$ with emphasis on dual polarization and multi-frequency SAR data is therefore timely.

Section 2 briefly recapitulates the fundamental results with some focus on dual polarization and multifrequency data. Section 3 gives a case study with single- and multi-frequency, full and dual polarimetry data. Section 4 discusses the results. Section 5 very briefly mentions software, and Section 6 gives conclusions.

The account given here closely follows that given our recent IEEE JSTARS paper. ${ }^{3}$

Image and Signal Processing for Remote Sensing XXI, edited by Lorenzo Bruzzone, Proc. of SPIE Vol. 9643, $964313 \cdot$ C 2015 SPIE · CCC code: 0277-786X/15/\$18 · doi: 10.1117/12.2194853 


\section{THEORY}

\subsection{Synthetic Aperture Radar}

A fully polarimetric SAR measures the 2 by 2 complex so-called scattering matrix at each resolution cell on the ground. The scattering matrix relates the incident and the scattered electric fields. ${ }^{5}$ If $S_{r t}$ denotes the complex scattering amplitude for receive and transmit polarization $(r, t \in\{h, v\}$ for horizontal and vertical polarization), then reciprocity, which normally applies to natural targets, gives $S_{h v}=S_{v h}$ (in the backscattering direction using the backscattering alignment convention). ${ }^{5}$ Assuming reciprocity, the scattering matrix may be represented by the three-component complex target vector $s=\left[S_{h h} S_{h v} S_{v v}\right]^{T}$, where ${ }^{T}$ denotes the transpose.

The inherent speckle in the SAR data can be reduced by spatial averaging at the expense of spatial resolution. In this so-called multi-look case a more appropriate representation of the backscattered signal is the covariance matrix in which the average properties of a group of resolution cells can be expressed in a single matrix formed by the outer products of the averaged target vectors. The average covariance matrix is defined as ${ }^{5}$

$$
\langle\boldsymbol{C}\rangle_{f u l l}=\left[\begin{array}{lll}
\left\langle S_{h h} S_{h h}^{*}\right\rangle & \left\langle S_{h h} S_{h v}^{*}\right\rangle & \left\langle S_{h h} S_{v v}^{*}\right\rangle \\
\left\langle S_{h v} S_{h h}^{*}\right\rangle & \left\langle S_{h v} S_{h v}^{*}\right\rangle & \left\langle S_{h v} S_{v v}^{*}\right\rangle \\
\left\langle S_{v v} S_{h h}^{*}\right\rangle & \left\langle S_{v v} S_{h v}^{*}\right\rangle & \left\langle S_{v v} S_{v v}^{*}\right\rangle
\end{array}\right]
$$

where $\langle\cdot\rangle$ denotes ensemble averaging and ${ }^{*}$ denotes complex conjugation. Reciprocity results in a covariance matrix with rank 3 .

Spaceborne instruments often transmit only one polarization, say horizontal, and receive both polarizations giving rise to dual polarization data. In this case we have the components $\left\langle S_{h h} S_{h h}^{*}\right\rangle,\left\langle S_{h h} S_{h v}^{*}\right\rangle$ and $\left\langle S_{h v} S_{h v}^{*}\right\rangle$ only. The resulting covariance matrix

$$
\langle\boldsymbol{C}\rangle_{d u a l}=\left[\begin{array}{ll}
\left\langle S_{h h} S_{h h}^{*}\right\rangle & \left\langle S_{h h} S_{h v}^{*}\right\rangle \\
\left\langle S_{h v} S_{h h}^{*}\right\rangle & \left\langle S_{h v} S_{h v}^{*}\right\rangle
\end{array}\right]
$$

has rank 2. (We could also transmit vertical polarization only and receive both or transmit and receive both.)

For both full and dual polarimetry the case where we have the diagonal elements of the covariance matrix only is also relevant.

\subsection{Test for Equality of Two Complex Covariance Matrices}

If the independent $p$ by $p$ Hermitian, positive definite matrices $\boldsymbol{X}$ and $\boldsymbol{Y}$ are complex Wishart distributed $\left(\boldsymbol{X}=n\langle\boldsymbol{C}\rangle_{x}\right.$ and $\boldsymbol{Y}=m\langle\boldsymbol{C}\rangle_{y}, n$ and $m$ are the number of looks), i.e., $\boldsymbol{X} \sim W_{C}\left(p, n, \boldsymbol{\Sigma}_{x}\right)$ with $\hat{\boldsymbol{\Sigma}}_{x}=\frac{1}{n} \boldsymbol{X}$ and $\boldsymbol{Y} \sim W_{C}\left(p, m, \boldsymbol{\Sigma}_{y}\right)$ with $\hat{\boldsymbol{\Sigma}}_{y}=\frac{1}{m} \boldsymbol{Y}$, then the likelihood ratio test statistic to test the so-called null hypothesis $H_{0}$ for equality of the two complex Wishart matrices

$$
H_{0}: \boldsymbol{\Sigma}_{x}=\boldsymbol{\Sigma}_{y}
$$

against all alternatives, is ${ }^{1}$ (results are also known for the real case ${ }^{6}$ )

$$
Q=\frac{(n+m)^{p(n+m)}}{n^{p n} m^{p m}} \frac{|\boldsymbol{X}|^{n}|\boldsymbol{Y}|^{m}}{|\boldsymbol{X}+\boldsymbol{Y}|^{n+m}}
$$

where $|\cdot|$ denotes the determinant; $Q \in[0,1]$ with $Q=1$ for equality. For the logarithm of $Q$ we get

$$
\begin{aligned}
\ln Q= & p[(n+m) \ln (n+m)-n \ln (n)-m \ln (m)] \\
& +n \ln |\boldsymbol{X}|+m \ln |\boldsymbol{Y}|-(n+m) \ln |\boldsymbol{X}+\boldsymbol{Y}| .
\end{aligned}
$$

If $n=m$ which is often the case we get

$$
\ln Q=n(2 p \ln 2+\ln |\boldsymbol{X}|+\ln |\boldsymbol{Y}|-2 \ln |\boldsymbol{X}+\boldsymbol{Y}|) .
$$




$$
\rho=1-\frac{2 p^{2}-1}{6 p}\left(\frac{1}{n}+\frac{1}{m}-\frac{1}{n+m}\right)
$$

and

$$
\omega_{2}=-\frac{p^{2}}{4}\left(1-\frac{1}{\rho}\right)^{2}+\frac{p^{2}\left(p^{2}-1\right)}{24 \rho^{2}}\left(\frac{1}{n^{2}}+\frac{1}{m^{2}}-\frac{1}{(n+m)^{2}}\right)
$$

and if the observed value of $-2 \rho \ln Q$ is $z=-2 \rho \ln q_{\mathrm{obs}}$, then the probability of finding a smaller value of $-2 \rho \ln Q$, i.e., the change probability, is

$$
P\{-2 \rho \ln Q \leq z\} \simeq P\left\{\chi^{2}\left(p^{2}\right) \leq z\right\}+\omega_{2}\left[P\left\{\chi^{2}\left(p^{2}+4\right) \leq z\right\}-P\left\{\chi^{2}\left(p^{2}\right) \leq z\right\}\right] .
$$

$1-P\{-2 \rho \ln Q \leq z\}=P\left\{Q<q_{\text {obs }}\right\}$ is the probability of equality, i.e., the no-change probability.

For full polarimetry data $p=3$, for dual polarimetry $p=2$, and for $\mathrm{HH}, \mathrm{HV}$ or VV data $p=1$.

\subsubsection{Block Diagonal Case}

Multi-frequency polarimetric data can also be described using the complex Wishart distribution. If $k$ is the number of polarimetric datasets acquired at different frequencies (and/or acquisition time points), and if the target vector data are available, the complex target vector is simply redefined as $s=\left[\begin{array}{llll}\boldsymbol{s}_{1}^{T} & \ldots & \boldsymbol{s}_{k}^{T}\end{array}\right]^{T}$ resulting in the average covariance matrix

$$
\langle\boldsymbol{C}\rangle=\left[\begin{array}{ccc}
\langle\boldsymbol{C}\rangle_{1} & \cdots & \langle\boldsymbol{C}\rangle_{1 k} \\
\vdots & \ddots & \vdots \\
\langle\boldsymbol{C}\rangle_{k 1} & \cdots & \langle\boldsymbol{C}\rangle_{k}
\end{array}\right]
$$

where $\langle\boldsymbol{C}\rangle_{i}$ is the average covariance matrix for acquisition $i$, and the elements of the complex matrices $\langle\boldsymbol{C}\rangle_{i j}, i \neq$ $j$, depend on the correlation between the acquisitions $\left(\langle\boldsymbol{C}\rangle_{i j}=\langle\boldsymbol{C}\rangle_{j i}^{*}\right)$. Again, the $p_{1}+\cdots+p_{k}$ by $p_{1}+\cdots+p_{k}$ Hermitian matrix $n\langle\boldsymbol{C}\rangle$ follows a complex Wishart distribution (where $n$ is number of looks). Often, however, the polarimetric SAR data from the acquisitions are already in the covariance matrix representation. In this case, the matrices $\langle\boldsymbol{C}\rangle_{i j}$ cannot be formed, and $n\langle\boldsymbol{C}\rangle$ does not follow a complex Wishart distribution, as some of the elements of the covariance matrix have been set to zero and we obtain a block diagonal $\langle\boldsymbol{C}\rangle$

$$
\langle\boldsymbol{C}\rangle=\left[\begin{array}{ccc}
\langle\boldsymbol{C}\rangle_{1} & \cdots & \mathbf{0} \\
\vdots & \ddots & \vdots \\
\mathbf{0} & \cdots & \langle\boldsymbol{C}\rangle_{k}
\end{array}\right]
$$

In this case we get the same test statistic as in the above cases (Equation 3) with $p=p_{1}+\cdots+p_{k}$. If

$$
\begin{aligned}
f_{i} & =p_{i}^{2} \\
f & =f_{1}+\cdots+f_{k} \\
\rho_{i} & =1-\frac{2 f_{i}-1}{6 p_{i}}\left(\frac{1}{n}+\frac{1}{m}-\frac{1}{n+m}\right) \\
\rho & =\frac{1}{f}\left(f_{1} \rho_{1}+\cdots+f_{k} \rho_{k}\right)
\end{aligned}
$$

and

$$
\omega_{2}=-\frac{f}{4}\left(1-\frac{1}{\rho}\right)^{2}+\frac{f_{1}\left(f_{1}-1\right)+\cdots+f_{k}\left(f_{k}-1\right)}{24 \rho^{2}}\left(\frac{1}{n^{2}}+\frac{1}{m^{2}}-\frac{1}{(n+m)^{2}}\right)
$$

then the probability of finding a smaller value of $-2 \rho \ln Q$ is the same as in Equation 4 with $p^{2}$ replaced by $f$ and with the immediately above values of $f, \rho$ and $\omega_{2}$. 


\subsubsection{Diagonal Only}

The diagonal only case for both full and dual polarization may be handled in a fashion similar to the block structure described in the previous section. ${ }^{1}$

\subsection{Practical Issues}

Here we deal with some computer implementation issues, namely the calculation of the determinants involved, and the calculation of probabilities. We also give examples on the handling of multi-frequency data based on the test statistic in Equation 3 and the probability measure in Equation 4 with associated values of $f, \rho$ and $\omega_{2}$.

\subsubsection{Determinants}

Let us write $\boldsymbol{Z}=n\langle\boldsymbol{C}\rangle$ as ( $n$ is the number of looks)

$$
\boldsymbol{Z}=n\left[\begin{array}{ccc}
\left\langle S_{h h} S_{h h}^{*}\right\rangle & \left\langle S_{h h} S_{h v}^{*}\right\rangle & \left\langle S_{h h} S_{v v}^{*}\right\rangle \\
\left\langle S_{h v} S_{h h}^{*}\right\rangle & \left\langle S_{h v} S_{h v}^{*}\right\rangle & \left\langle S_{h v} S_{v v}^{*}\right\rangle \\
\left\langle S_{v v} S_{h h}^{*}\right\rangle & \left\langle S_{v v} S_{h v}^{*}\right\rangle & \left\langle S_{v v} S_{v v}^{*}\right\rangle
\end{array}\right]=\left[\begin{array}{ccc}
k & a & \rho \\
a^{*} & \xi & b \\
\rho^{*} & b^{*} & \zeta
\end{array}\right] .
$$

This gives the determinant

$$
|\boldsymbol{Z}|=k \xi \zeta+a b \rho^{*}+\rho a^{*} b^{*}-|\rho|^{2} \xi-|b|^{2} k-|a|^{2} \zeta .
$$

The first, fourth, fifth and sixth terms are real. The second and third terms are complex and each others conjugate

$$
\begin{aligned}
a b \rho^{*} & =\left(a_{R}+i a_{I}\right)\left(b_{R}+i b_{I}\right)\left(\rho_{R}-i \rho_{I}\right) \\
& =\rho_{R}\left(a_{R} b_{R}-a_{I} b_{I}\right)+\rho_{I}\left(a_{I} b_{R}+a_{R} b_{I}\right)+i\left[\rho_{R}\left(a_{I} b_{R}+a_{R} b_{I}\right)-\rho_{I}\left(a_{R} b_{R}-a_{I} b_{I}\right)\right] \\
a^{*} b^{*} \rho & =\left(a_{R}-i a_{I}\right)\left(b_{R}-i b_{I}\right)\left(\rho_{R}+i \rho_{I}\right) \\
& =\rho_{R}\left(a_{R} b_{R}-a_{I} b_{I}\right)+\rho_{I}\left(a_{I} b_{R}+a_{R} b_{I}\right)-i\left[\rho_{R}\left(a_{I} b_{R}+a_{R} b_{I}\right)-\rho_{I}\left(a_{R} b_{R}-a_{I} b_{I}\right)\right] .
\end{aligned}
$$

Since the imaginary parts cancel out, the sum of the two terms is real and equal to twice the real part.

\subsubsection{Probabilities}

Rewriting the approximate probability of finding a smaller value of $-2 \rho \ln Q$ in Equation 4, the probabilities involved can be found by two calls to the incomplete gamma function, $\gamma\left(\frac{\nu}{2}, \frac{X^{2}}{2}\right)^{7,8}$ where $\nu$ is the number of degrees of freedom and $X^{2}$ is the test statistic, ${ }^{1,3}$

$$
P\{-2 \rho \ln Q \leq z\} \simeq\left[1-\omega_{2}\right] \gamma\left(\frac{f}{2},-\rho \ln Q\right)+\omega_{2} \gamma\left(\frac{f}{2}+2,-\rho \ln Q\right) .
$$

\subsubsection{Bi-Frequency Data}

Let us consider for example fully polarimetric C- and L-band data given as covariance data, i.e., we do not have access to the complex target vectors themselves, only their outer products. Here we can form the joint covariance matrix multiplied by $n$, see Equations 1, 5 and 6 ,

$$
\boldsymbol{Z}=\left[\begin{array}{cccccc}
k_{C} & a_{C} & \rho_{C} & 0 & 0 & 0 \\
a_{C}^{*} & \xi_{C} & b_{C} & 0 & 0 & 0 \\
\rho_{C}^{*} & b_{C}^{*} & \zeta_{C} & 0 & 0 & 0 \\
0 & 0 & 0 & k_{L} & a_{L} & \rho_{L} \\
0 & 0 & 0 & a_{L}^{*} & \xi_{L} & b_{L} \\
0 & 0 & 0 & \rho_{L}^{*} & b_{L}^{*} & \zeta_{L}
\end{array}\right]
$$

In this case $k=2, p_{1}=p_{2}=3, p=6$ and $f=18$. For number of looks equal to 13 (as in the examples below) $\rho=0.8910$ and $\omega_{2}=0.0109$. The determinant equals the product of the two determinants of each block matrix, see Equation 7. 
In the same situation for dual polarization $\mathrm{C}$ - and L-band data, say, $\mathrm{HH}$ and $\mathrm{HV}$, we get the joint covariance matrix multiplied by $n$, see Equations 2, 5 and 6 ,

$$
\boldsymbol{Z}=\left[\begin{array}{cccc}
k_{C} & a_{C} & 0 & 0 \\
a_{C}^{*} & \xi_{C} & 0 & 0 \\
0 & 0 & k_{L} & a_{L} \\
0 & 0 & a_{L}^{*} & \xi_{L}
\end{array}\right]
$$

In this case $k=2, p_{1}=p_{2}=2, p=4$ and $f=8$. For number of looks equal to 13 (as in the examples below) $\rho=0.9327$ and $\omega_{2}=0.0015$. Again the determinant equals the product, here

$$
|\boldsymbol{Z}|=\left(k_{C} \xi_{C}-\left|a_{C}\right|^{2}\right)\left(k_{L} \xi_{L}-\left|a_{L}\right|^{2}\right) .
$$

\section{CASE STUDY}

Full polarimetry, C- and L-band EMISAR ${ }^{9,10}$ data from the following dates

- 20 May 1998 (called C64 and L64),

- 16 June 1998 (called C65 and L65),

are used. The images have 5 meter pixels, 1024 lines and 1024 samples. Number of looks is 13 ( $n$ and $m$ above).

Figures 1 to 4 show RGB combinations of the diagonal elements, i.e., the backscatter coefficients, of the full polarimetry covariance matrix for C64, C65, L64 and L65, respectively. The two C-band images are stretched identically and so are the two L-band images.

\subsection{Bi-Temporal Bi-Frequency Change Detection, Full Polarimetry}

Here we use the data C64/L64 and C65/L65 in a joint analysis as sketched in Section 2.3.3 to test for change in the bi-frequency, bi-temporal data.

First, Figures 5 and 7 show $-2 \rho \ln Q$ for C-band and L-band alone (stretched between 0 and 50). Figures 6 and 8 show the corresponding probabilities thresholded at 0.9999. In Figures 6 and 8 "B" marks a beets field, "P" marks pea fields, "W" winter barley fields, and "G" a grass field. We have no in situ information on the other highlighted but unmarked fields in the images.

Figures 9 and 10 show $-2 \rho \ln Q$ (stretched between 0 and 100) and $P$ (the probability of finding a smaller value of $-2 \rho \ln Q$ ) thresholded at 0.9999 for full polarimetry C64/L64 vs C65/L65.

\subsection{Bi-Temporal Change Detection, Dual Polarimetry}

As an example we here use the HH/HV data L64 and L65 to test for change in the bi-temporal data.

Figures 11 and 12 show $-2 \rho \ln Q$ (stretched between 0 and 50) and $P$ thresholded at 0.9999 for dual polarimetry L64 vs L65, HH/HV.

\section{DISCUSSION}

Wooded areas surrounding some of the large fields in the middle of the images and along the lake and which exhibit no change between the two acquisitions, are seen in cyan in Figures 1 to 4 . Tables 1 and 2 show average backscatter coefficients, correlation and phase difference between HH and VV over a small wooded area and the beet field (see Figures 6 and 8) for C- and L-band data. Also they show the average no-change probability associated with the test statistic. For the bi-frequency analysis $P\left\{Q<q_{\mathrm{obs}}\right\}$ is 0.4893 for the wooded area and 0.0005 for the beet field. The wooded areas are all coniferous forests, and hence no change is occurring between May and June, in correspondence with the results of the change detector at all frequency combinations. The beets field, on the other hand, is changing from a bare field in May to a field with some leaves developed in June, and hence, changes are detected at all frequency combinations. 
Table 1. Average polarimetric parameters and no-change probability over a wooded area for C- and L-band data.

\begin{tabular}{|l|r|r|r|r|}
\hline & C May & C Jun & L May & L Jun \\
\hline$\sigma_{h h}^{0}[\mathrm{~dB}]$ & -6.5 & -6.1 & -5.4 & -5.4 \\
\hline$\sigma_{h v}^{0}[\mathrm{~dB}]$ & -12.7 & -12.2 & -10.6 & -10.6 \\
\hline$\sigma_{v v}^{0}[\mathrm{~dB}]$ & -7.2 & -6.7 & -7.1 & -7.3 \\
\hline$\rho$ & 0.49 & 0.48 & 0.38 & 0.41 \\
\hline$\phi_{h h-v v}[\mathrm{rad}]$ & -0.16 & -0.08 & -0.11 & -0.17 \\
\hline$P\left\{Q<q_{\text {obs }}\right\}$ & & 0.4850 & & 0.4913 \\
\hline
\end{tabular}

Table 2. Average polarimetric parameters and no-change probability over the beet field for C- and L-band data.

\begin{tabular}{|l|r|r|r|r|}
\hline & C May & C Jun & L May & L Jun \\
\hline$\sigma_{h h}^{0}[\mathrm{~dB}]$ & -16.1 & -10.3 & -24.6 & -13.1 \\
\hline$\sigma_{h v}^{0}[\mathrm{~dB}]$ & -27.4 & -17.4 & -35.0 & -24.6 \\
\hline$\sigma_{v v}^{0}[\mathrm{~dB}]$ & -16.1 & -11.2 & -22.4 & -15.3 \\
\hline$\rho$ & 0.81 & 0.54 & 0.80 & 0.57 \\
\hline$\phi_{h h-v v}[\mathrm{rad}]$ & 0.21 & 0.12 & 0.40 & 1.11 \\
\hline$P\left\{Q<q_{\text {obs }}\right\}$ & & 0.0015 & & 0.0004 \\
\hline
\end{tabular}

Changes are detected in the winter barley fields between May and June for C-band and the C- and L-band combinations, but not for the L-band alone. From May to June, the head of the winter barley starts to hang down, and hence due to such an angle of the head and size of the head relative to the wavelength at C-band it generates cross-polarized backscatter in June. ${ }^{11}$ This change in the backscatter causes a detected change from May to June in C-band, whereas the size of the head is small compared to the wavelength at L-band, and hence, it does not generate a change in the backscatter at L-band.

The peas fields develop from a stage of some leaves developed in May with a height of approximately 15 $\mathrm{cm}$ to a stage of emergence in June with heights from 40-60 cm. At C-band, the backscatter is dominated by volume scattering at both acquisitions, and hence no changes are seen in the backscatter from the fields. The large change in biomass between the two acquisitions causes a change in the L-band backscatter, and a change is detected at L-band and also for the combination of C- and L-band.

The grass field shows a detected change from May to June at C-band, and also in the combination of C- and L-band. The grass changes from standing relatively straight up in May, to lying down in June. This change causes an increase in the cross-polarized backscatter at C-band, and hence the detection of the change at Cband. At L-band, on the other hand, the change is not as dramatic, because the L-band backscatter is more sensitive to the total biomass, which is not changing so much. At L-band, the backscatter in May includes some double-bounce scattering, whereas this component is smaller in June, where the grass is more randomly oriented. Some change is detected using full polarimetry at L-band as seen in Figure 8, whereas no change is detected using dual polarimetry $(\mathrm{HH} / \mathrm{HV})$, which is not sensitive to the double-bounce scattering.

It is clear from the above results, that the combination of C- and L-band for the change detection captures all changes that are detected by either C- or L-band.

\section{SOFTWARE}

Matlab code to perform the analysis is described elsewhere ${ }^{3}$ and is available on Allan Nielsen's homepage. Code in ENVI/IDL and Python for the single frequency methods is available on Dr. Mort Canty's homepage and described in his textbook. ${ }^{12}$ 


\section{CONCLUSIONS}

Change in winter barley fields and in a grass field is detected in C-band and not in L-band. Change in pea fields is detected in L-band and not in C-band. The multi-frequency analysis detects change in both winter barley, the grass field, and the pea fields. Change in a beets field is detected in both C- and L-band as well as in the multi-frequency analysis.

The results obtained with the change detector are confirmed by the change and absence of change in the polarimetric parameters over the beet field and a wooded area, respectively.

\section{REFERENCES}

[1] Conradsen, K., Nielsen, A. A., Schou, J., and Skriver, H., "A test statistic in the complex Wishart distribution and its application to change detection in polarimetric SAR data," IEEE Transactions on Geoscience and Remote Sensing 41, 4-19 (Jan. 2003). http://www.imm.dtu.dk/pubdb/p.php?1219.

[2] Schou, J., Skriver, H., Nielsen, A. A., and Conradsen, K., "CFAR edge detector for polarimetric SAR images," IEEE Transactions on Geoscience and Remote Sensing 41, 20-32 (Jan. 2003). http://www.imm.dtu.dk/pubdb/p.php?1224.

[3] Nielsen, A. A., Conradsen, K., and Skriver, H., "Change detection in full and dual polarization, single- and multi-frequency SAR data," IEEE Journal of Selected Topics in Applied Earth Observations and Remote Sensing 8, 4041-4048 (Aug. 2015). http://www.imm.dtu.dk/pubdb/p.php?6827.

[4] Akbari, V., Anfinsen, S. N., Doulgeris, A. P., and Eltoft, T., "The Hotelling-Lawley trace statistic for change detection in polarimetric SAR data under the complex Wishart distribution," in IEEE IGARSS, 4162-4165 (21-26 July 2013).

[5] van Zyl, J. J. and Ulaby, F. T., "Scattering matrix representation for simple targets," in Radar Polarimetry for Geoscience Applications, Ulaby, F. T. and Elachi, C., eds., Artech, Norwood, MA (1990).

[6] Anderson, T. W., An Introduction to Multivariate Statistical Analysis, John Wiley, New York, third ed. (2003).

[7] Abramowitz, M. and Stegun, I. A., Handbook of Mathematical Functions, National Bureau of Standards (1964).

[8] Press, W. H., Flannery, B. P., Teukolsky, S. A., and Vetterling, W. T., Numerical Recipes in C: The Art of Scientific Computing, Cambridge University Press, second ed. (1992).

[9] Madsen, S. N., Christensen, E. L., Skou, N., and Dall, J., "The Danish SAR system: Design and initial tests," IEEE Transactions on Geoscience and Remote Sensing 29, 417-476 (1991).

[10] Christensen, E. L., Skou, N., Dall, J., Woelders, K., rgensen, J. H. J., Granholm, J., and Madsen, S. N., "EMISAR: An absolutely calibrated polarimetric L- and C-band SAR," IEEE Transactions on Geoscience and Remote Sensing 36, 1852-1865 (1998).

[11] Skriver, H., Svendsen, M. T., and Thomsen, A. G., "Multitemporal L- and C-band polarimetric signatures of crops," IEEE Transactions on Geoscience and Remote Sensing 37, 2413-2429 (1999).

[12] Canty, M. J., Image Analysis, Classification and Change Detection in Remote Sensing. With Algorithms for ENVI/IDL and Python, Taylor \& Francis, CRC Press, third ed. (2014). 


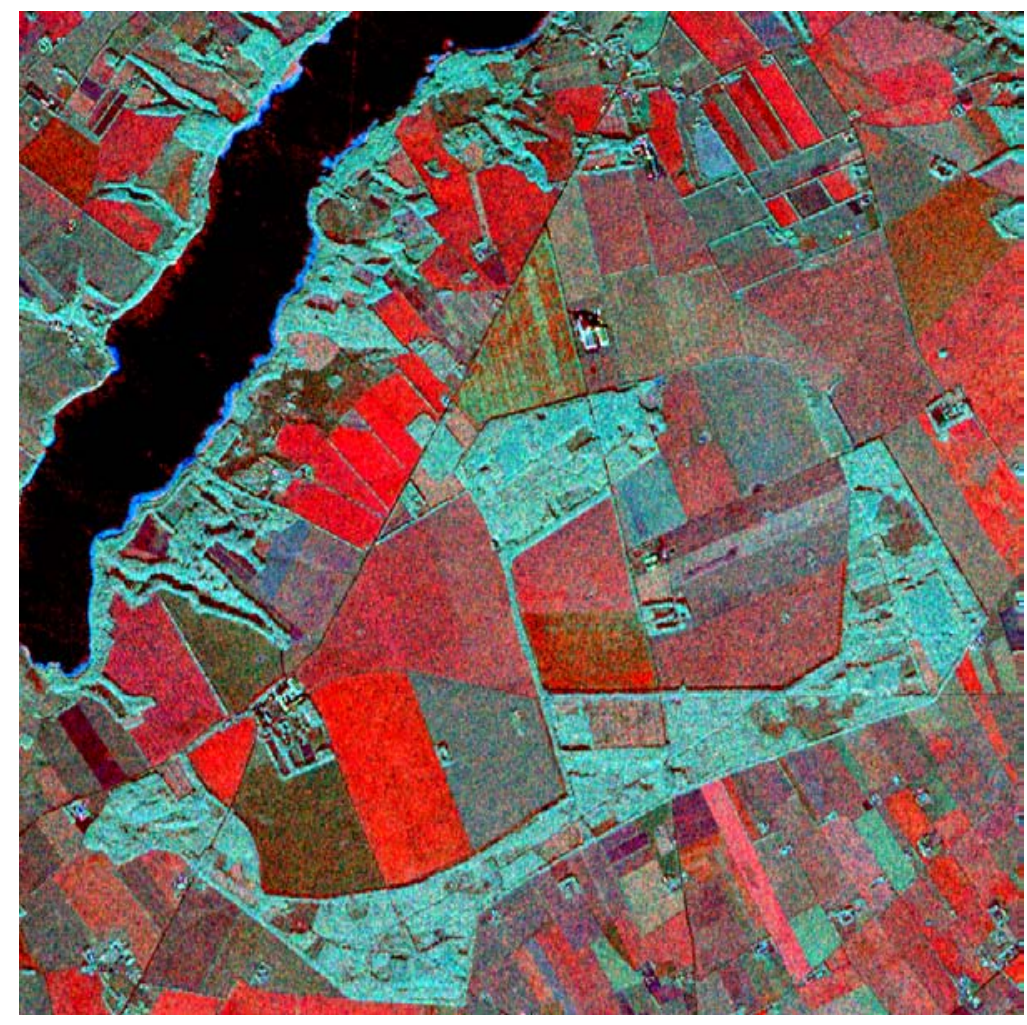

Figure 1. EMISAR C-band data C64 20 May 1998, Pauli RGB.

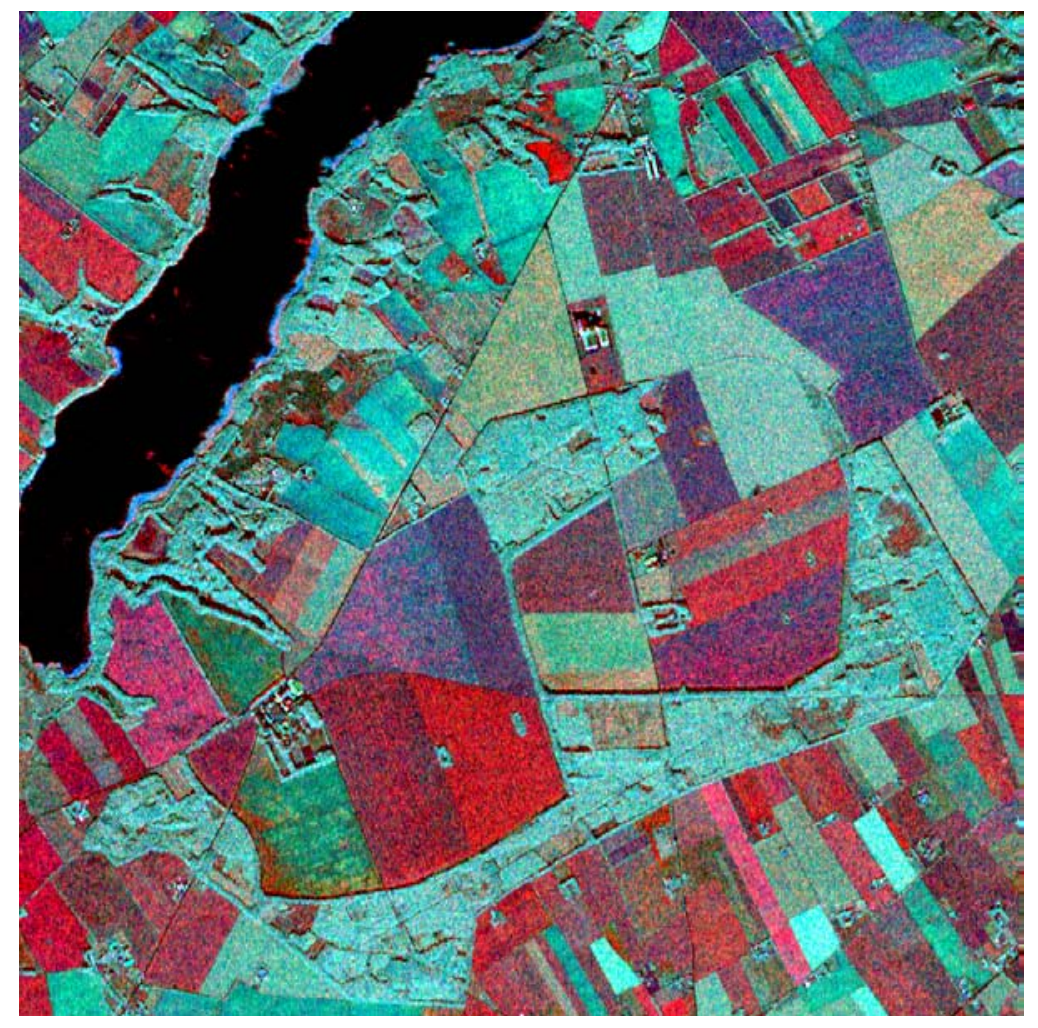

Figure 2. EMISAR C-band data C65 16 June 1998, Pauli RGB. 


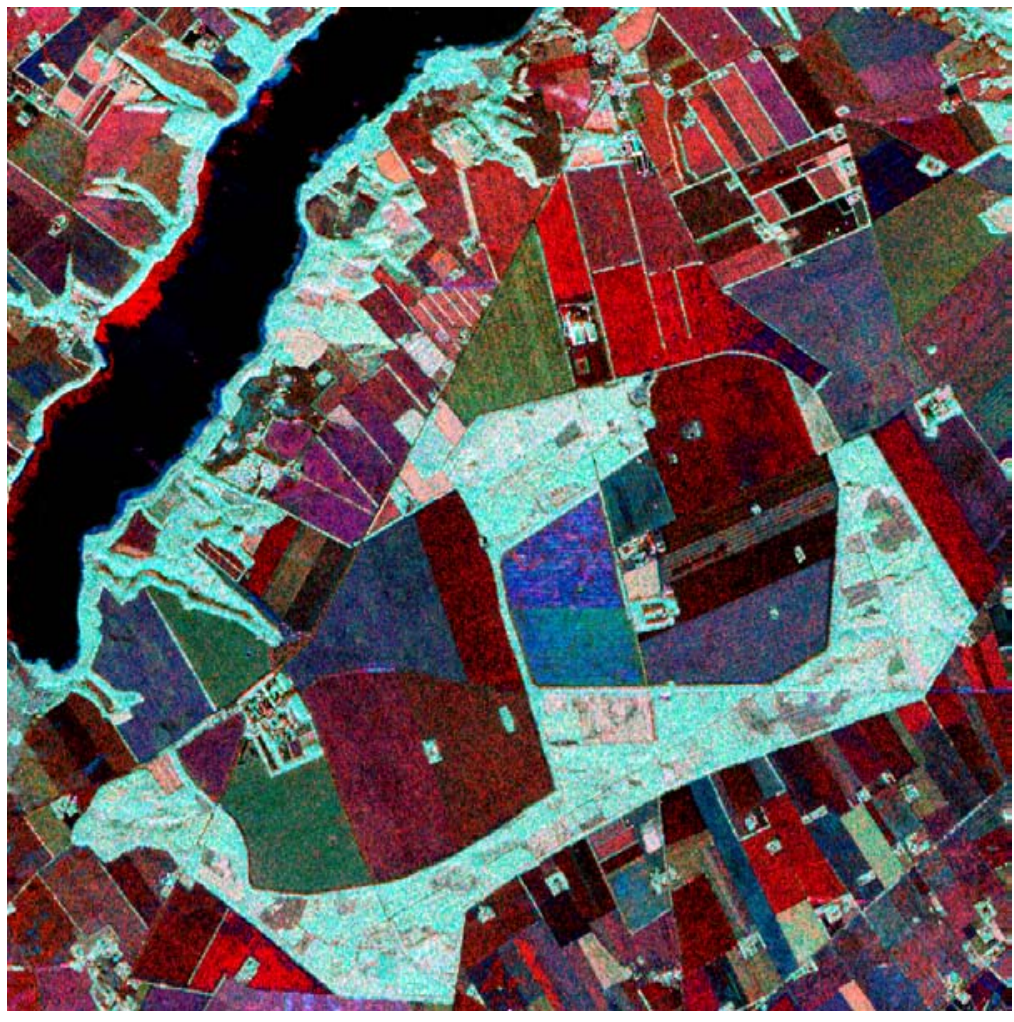

Figure 3. EMISAR L-band data L64 20 May 1998, Pauli RGB.

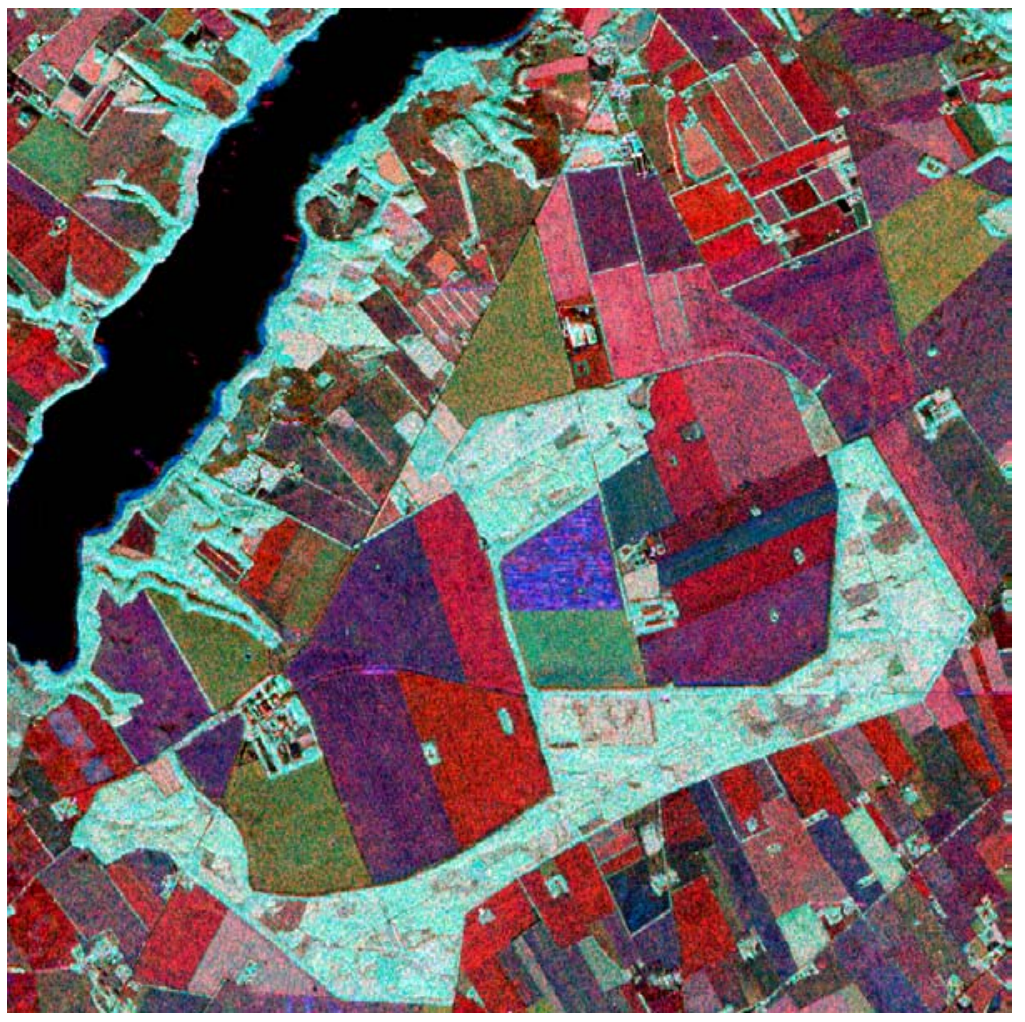

Figure 4. EMISAR L-band data L65 16 June 1998, Pauli RGB. 


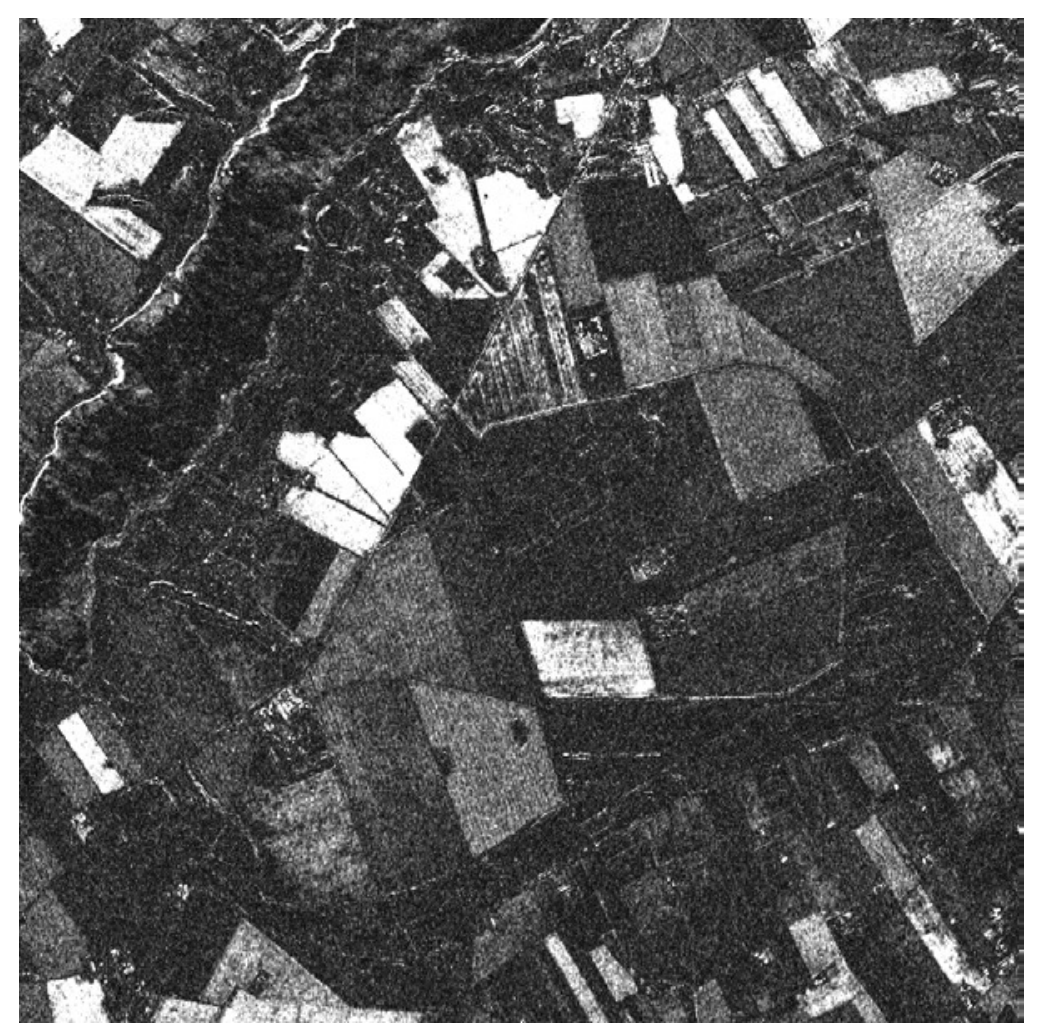

Figure 5. $-2 \rho \ln Q$ for C64 vs C65, full polarimetry.

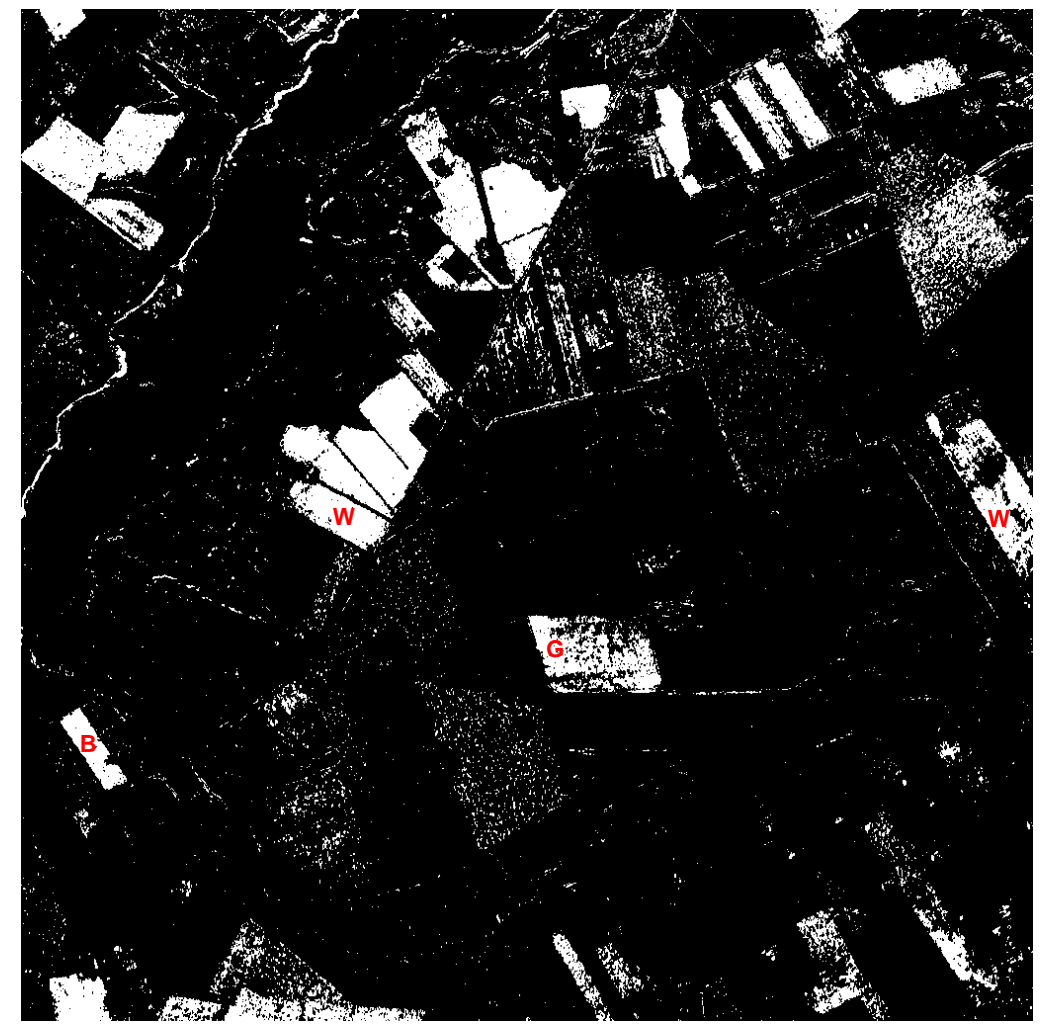

Figure 6. $P\{-2 \rho \ln Q \leq z\}$ thresholded at 0.9999 for $\mathrm{C} 64$ vs $\mathrm{C} 65$, full polarimetry. "B" marks a beets field, "W" marks winter barley fields. "G" marks a grass field. 


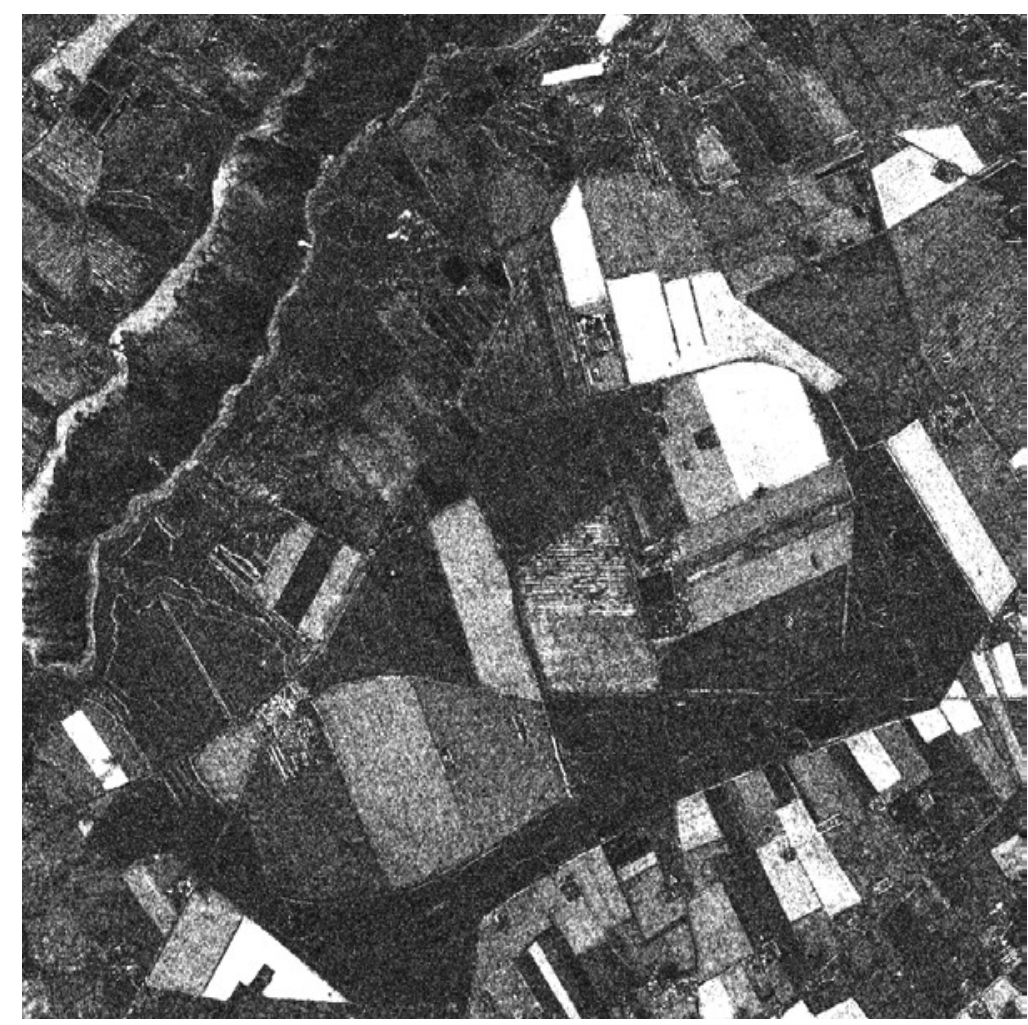

Figure 7. $-2 \rho \ln Q$ for L64 vs L65, full polarimetry.

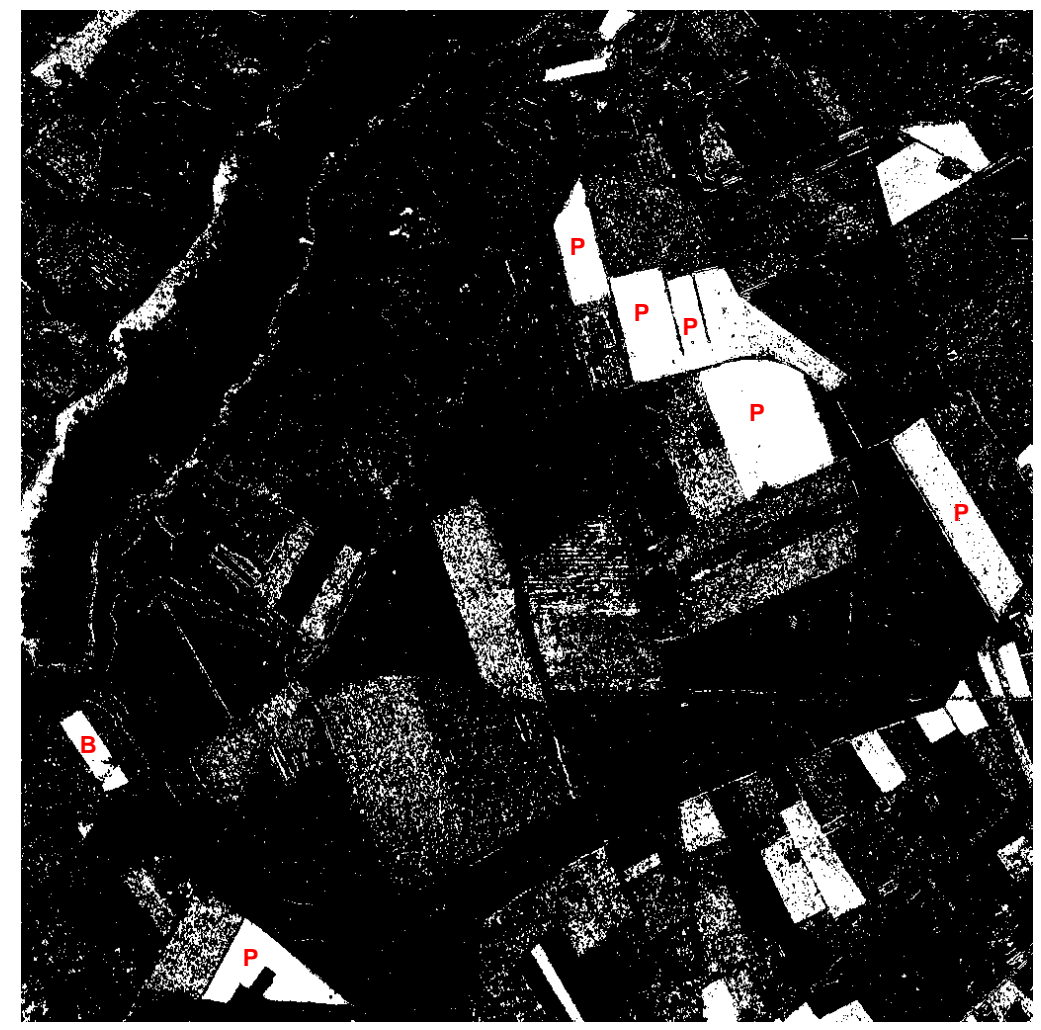

Figure 8. $P\{-2 \rho \ln Q \leq z\}$ thresholded at 0.9999 for L64 vs L65, full polarimetry. "B" marks a beets field, "P" marks pea fields. 


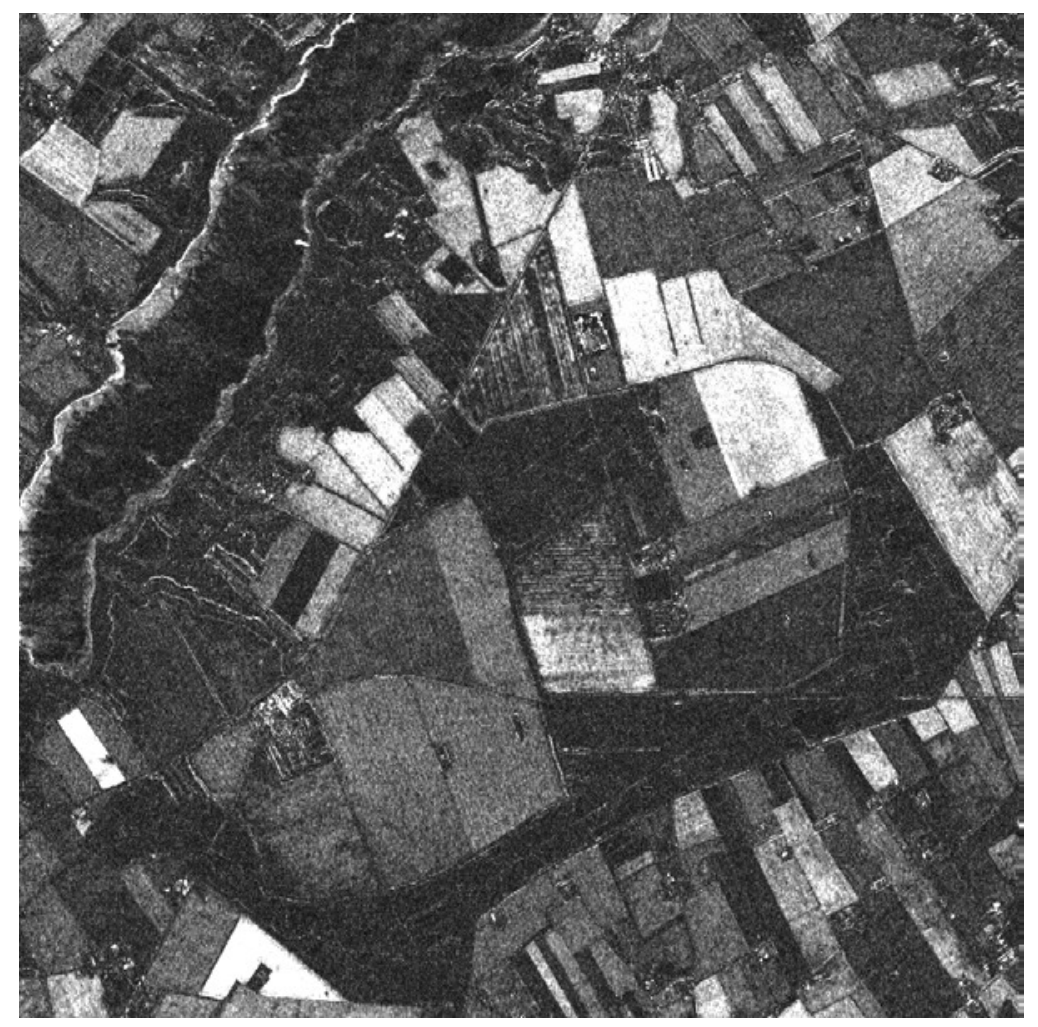

Figure 9. $-2 \rho \ln Q$ for C64/L64 vs C65/L65, full polarimetry.

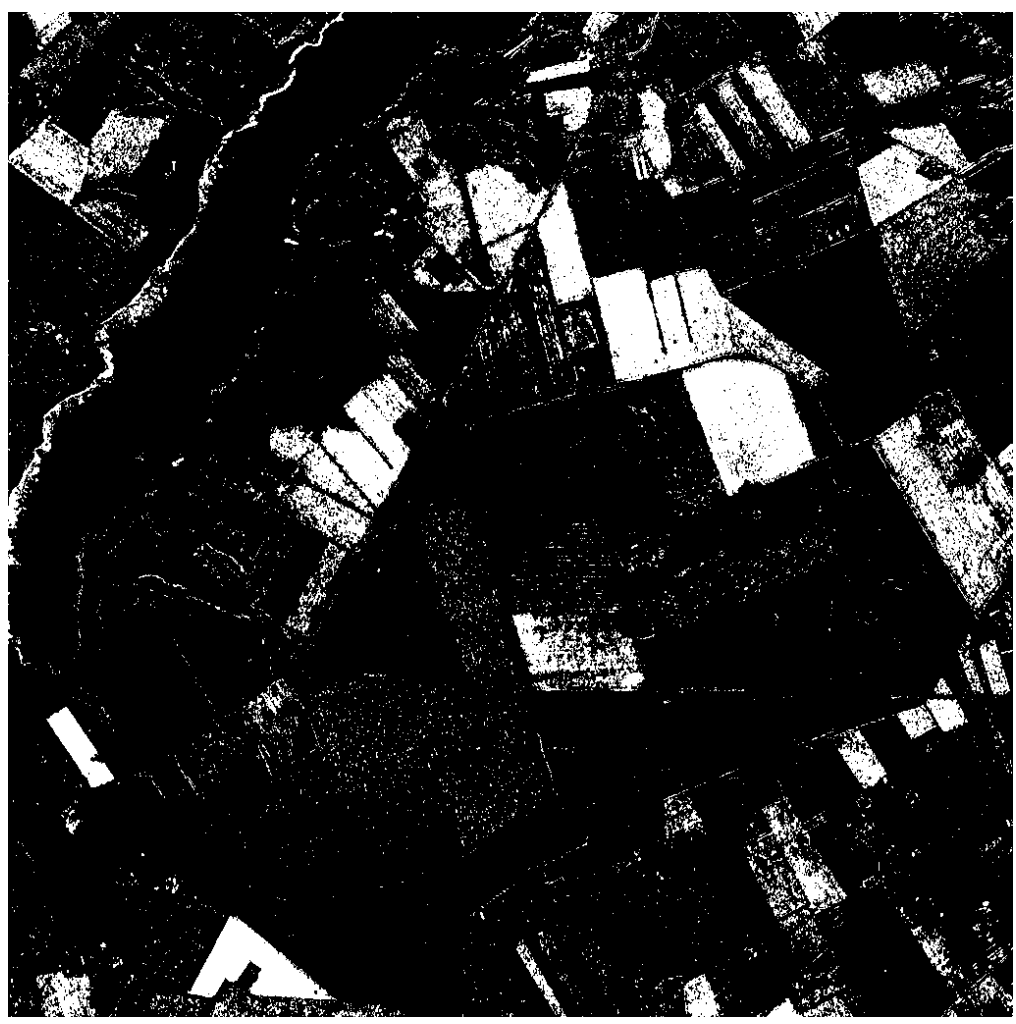

Figure 10. $P\{-2 \rho \ln Q \leq z\}$ thresholded at 0.9999 for C64/L64 vs C65/L65, full polarimetry. 


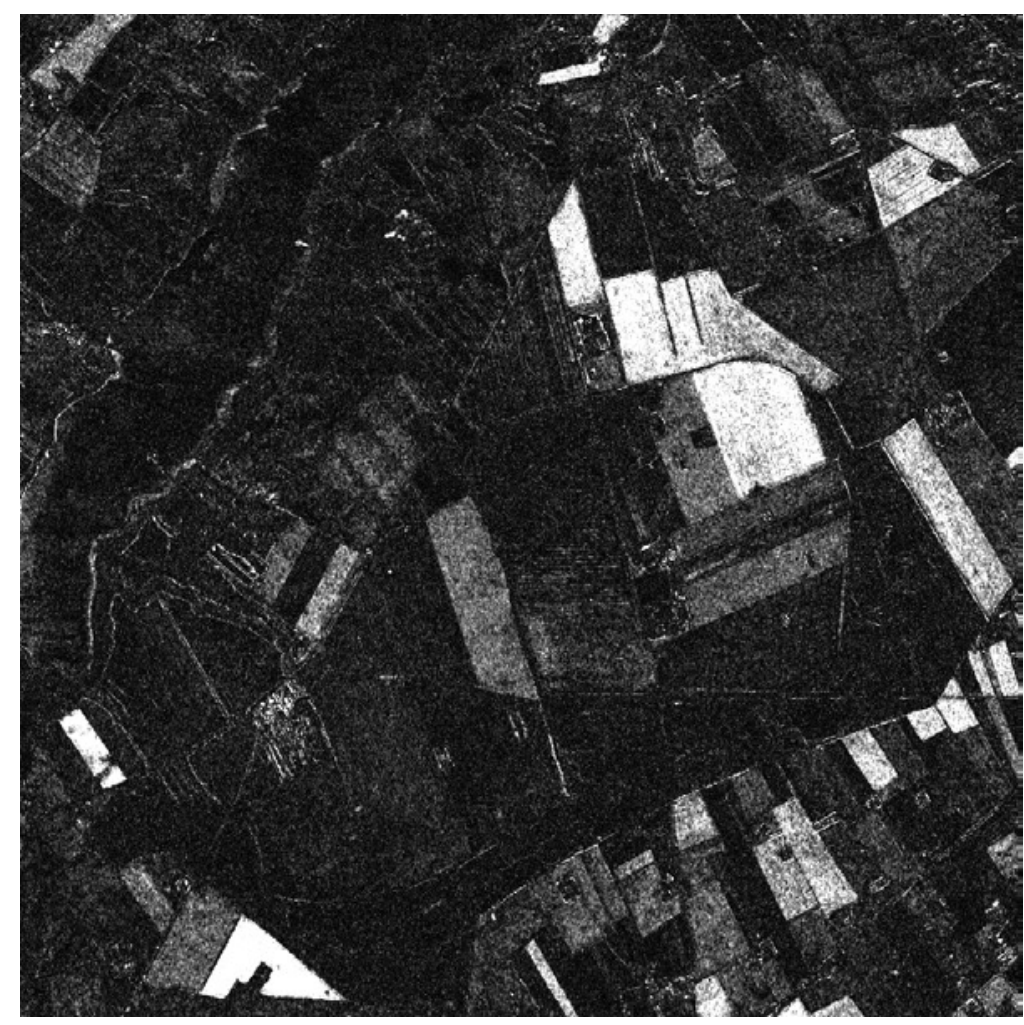

Figure 11. $-2 \rho \ln Q$ for L64 vs L65, dual polarimetry $\mathrm{HH} / \mathrm{HV}$.

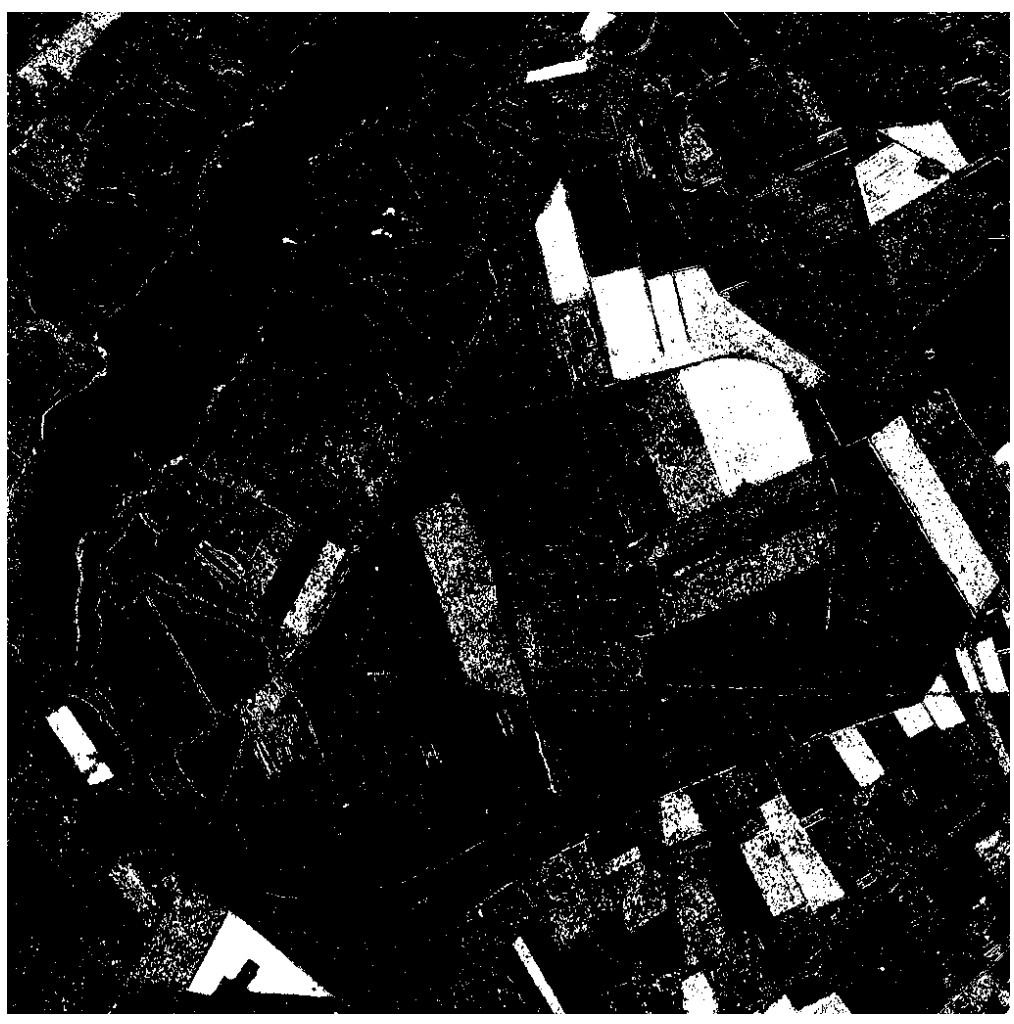

Figure 12. $P\{-2 \rho \ln Q \leq z\}$ thresholded at 0.9999 for L64 vs L65, dual polarimetry HH/HV. 